\title{
Telomerase expression in amyotrophic lateral sclerosis (ALS) patients
}

\author{
Bruna De Felice ${ }^{1}$, Anna Annunziata ${ }^{2}$, Giuseppe Fiorentino ${ }^{2}$, Francesco Manfellotto ${ }^{1}$, Raffaella D'Alessandro ${ }^{1}$, \\ Rita Marino ${ }^{3}$, Marco Borra ${ }^{3}$ and Elio Biffali ${ }^{3}$ \\ Telomerase and telomeric complex have been linked to a variety of disease states related to neurological dysfunction. In \\ amyotrophic lateral sclerosis (ALS) patients, telomerase activity, as human telomerase reverse transcriptase (hTERT) expression, \\ has not been characterized yet. Here, for the first time, we characterized telomerase and related pathway in blood sample and \\ spinal cord from ALS patients compared with healthy controls. We found that hTERT expression level was significantly lower in \\ ALS patients and was correlated either to p53 mRNA expression or p21 expression, pointing out the hypothesis that telomerase \\ inhibition could be a pathogenetic contributor to neurodegeneration in ALS. As a consequence of the reduced telomerase \\ activity, we identified shorter telomeres in leukocytes from sporadic ALS patients compared with healthy control group. \\ Journal of Human Genetics (2014) 59, 555-561; doi:10.1038/jhg.2014.72; published online 21 August 2014
}

\section{INTRODUCTION}

Telomerase is required for complete replication of telomeres as it is the specialized enzyme capable of reverse transcribing DNA. In humans, the core enzyme comprises an RNA component, termed hTR or hTERC (Telomerase RNA Component), that contains a template region that is complementary to the human telomere sequence and the protein human telomerase reverse transcriptase (hTERT) that contains a reverse transcriptase domain able to catalyze the addition of a six-nucleotide repeat to the ends of telomeres. ${ }^{1}$

Telomerase expression is low or absent in normal human somatic cells, but highly expressed in embryonic cells, adult male germ cells, in stem cells of proliferating tissues and in over $90 \%$ of human malignancies. ${ }^{2}$ In malignant cells, hTR and hTERT are significantly upregulated compared with normal cell counterparts. ${ }^{3}$

Regulation of telomerase is very complex as many aspects can effect hTERT expression and telomerase activity. Telomerase may be regulated through transcriptional regulation, posttranscriptional regulation, posttranslational regulation, methylation of the hTERT promoter and telomeric regions, the possible role of noncoding RNAs including microRNAs and sirtuins and TERRA (telomeric repeat-containing RNA).

The tumor suppressor gene p53 is a sequence-specific transcription factor that can mediate many downstream effects such as growth arrest and apoptosis through activation or repression of its target genes. ${ }^{4}$ In several cancer cell lines, overexpression of wild-type p53 downregulates telomerase independently of its effects on apoptosis, as transcriptional repression of hTERT by wild-type p53 is preceded by downregulation of hTERT messenger RNA (mRNA). ${ }^{5}$ The p53 represses telomerase in a cell type-specific manner occurring through an indirect mechanism that is mediated by the $\mathrm{p} 21 / \mathrm{E} 2 \mathrm{~F}$ pathway and involves recruitment of histone deacetylase-containing pocket protein complexes. These results contribute to our understanding of the relationship between p53 and telomerase and provide clues for elucidation of the mechanisms of p53-mediated transcriptional repression. The hTERT has long been associated with oncoproteins such as p21 and p53 owing to the link between telomerase activity and such proteins. ${ }^{6-9}$

In addition to the conventional role of telomerase in telomere synthesis, an increasing number of studies have indicated that telomerase has a regulatory role in gene expression. Indeed, it has been shown that expression of hTERT in human mammary epithelial cells resulted in the upregulation of five growth-promoting genes, and the downregulation of seven growth-inhibitory genes. ${ }^{10}$ Even if the systems by which telomerase participates in gene expression are largely unknown, one theory suggests that telomerase may be involved in epigenetic modifications or the modulation of chromatin structure that indirectly affect gene expression. ${ }^{11}$

Telomeres and telomerase have largely been investigated for their roles in cancer cellular senescence in mitotic cells.

Widespread work has shown that telomerase dysfunction and/or telomere shortening play an important role in the development of the lung disease idiopathic pulmonary fibrosis, dyskeratosis congenita and aplastic anemia. ${ }^{12}$ Beside, recent studies have linked dysfunction of the telomeric complex to a variety of disease states related to neurological disfunction. ${ }^{13}$ Transgenic animal models have shed light on the role of the telomeric complex in normal central nervous

${ }^{1}$ DISTABIF, Department of Environmental, Biological and Pharmaceutical Sciences and Technologies, University of Naples II, Caserta, Italy; ${ }^{2}$ Division of Respiratory Physiopathology, Monaldi Hospital, Naples, Italy and 'Stazione Zoologica 'Anton Dohrn', Naples, Italy

Correspondence: Professor B De Felice, DISTABIF, Department of Environmental, Biological and Pharmaceutical Sciences and Technologies, University of Naples II, Via Vivaldi 43, 81100 Caserta, Italy.

E-mail: bruna.defelice@unina2.it

Received 5 March 2014; revised 13 July 2014; accepted 17 July 2014; published online 21 August 2014 
system function and development. Knock-in allele for TERT mice showed no detectable telomerase and demonstrated reduced brain weights, lower numbers of mature oligodendrocytes and dysfunctional myelination. ${ }^{14}$ A neuroprotective function of TERT has been demonstrated in an experimental model relevant to Alzheimer's disease, suggesting the possibility that restoration of TERT expression in neurons, in the adult brain, may protect against age-related neurodegeneration. ${ }^{15}$

Telomerase activity and hTERT expression in amyotrophic lateral sclerosis (ALS) patients have not been characterized yet. ALS is a terminal disease involving the progressive degeneration of motor neurons within the motor cortex, brainstem and spinal cord. Most cases are sporadic ALS (sALS) with unknown causes, suggesting that the etiology of sALS may not be limited to the genotype of patients, but may be influenced by the interaction of other genetic and epigenetic factors. ${ }^{16}$

In a previous study, in adult mesenchymal stem cells derived from ALS patients, telomerase activity and telomerase mRNA transcripts have been characterized, showing that telomerase activity was 106-fold lower compared with glioblastoma tumor cells; however, telomerase activity was not compared with healthy controls. ${ }^{17}$

Interestingly, it has been shown that a novel compound, AGS-499, increases telomerase activity and expression in the mouse brain and spinal cord, delaying the onset and progression of the ALS disease in SOD1 transgenic mice (which exhibit the histopathological features observed in sporadic and familial ALS), and increasing the survival of motor neurons in the spinal cord by $60 \% .{ }^{17}$ Therefore, such data could suggest a lack of telomerase activity and hTERT expression in ALS patients.

In the present research, we aimed to characterize telomerase and related pathway in blood sample and spinal cord from sALS patients compared with healthy controls, pointing out the hypothesis that telomerase inhibition could be a pathogenetic contributor to neurodegeneration in ALS.

\section{MATERIALS AND METHODS}

Ethics statement

We obtained ethics approval for our study from the ethics committee (also known as an Institutional Review Board) at our institution. All the participants, even if they were over 60 , had the capacity to consent and we obtained written informed consent from all participants involved in the study.

\section{Clinical samples}

Two groups of patients were enrolled here (see Tables 1 and 2, for patient characteristics) in Monaldi Hospital, Naples, Italy.

The peripheral blood leukocytes were obtained from a first group of sALS patients and the unaffected controls. The group included 50 patients and 50

Table 1 Group 1 patient characteristics from Monaldi Hospital, Naples, Italy

\begin{tabular}{lcc}
\hline Characteristics & SALS patients $(\mathrm{n}=50)$ & Controls $(\mathrm{n}=50)$ \\
\hline Age (years) & $61.23 \pm 9.5$ & $61.2 \pm 9.8$ \\
Gender & & \\
$\quad$ Males $(n)$ & 27 & 26 \\
$\quad$ Females $(n)$ & 23 & 24 \\
Mean length of the disease (months) & $12.66 \pm 6.1$ & - \\
Onset of the disease (\%) & & - \\
$\quad$ Bulbar & $32 \%$ & - \\
Limb & $68 \%$ & - \\
\hline
\end{tabular}

Abbreviation: SALS, sporadic amyotrophic lateral sclerosis. unaffected healthy controls. The mean age of patients and controls was $61.2 \pm 9$ years (Table 1).

Spinal cord was obtained from a second group of 10 sALS patients and 10 unaffected healthy controls. The mean age of patients and controls was $62.2 \pm 8$ years (Table 2 ).

All patients were diagnosed as clinically definite sALS with the support of electroneuromyography. The onset of the disease was 1 to 2 years before diagnosis.

\section{RNA extraction from blood and spinal cord samples from sALS patients}

Ten micrograms of total RNA was obtained from peripheral blood samples that were drawn from patients and controls in the morning. For isolation and purification of RNA from whole blood, Trizol (Invitrogen, Dublin, Ireland) method has been used for all samples within $1 \mathrm{~h}$ from drawing, thus reducing RNA degradation. RNA was isolated including an optional DNase digestion step.

Spinal cord from sALS cases and neuropathologically normal individuals matched for age was obtained by post-mortem autopsy and stored at $-80^{\circ} \mathrm{C}$ until total RNA isolation by Trizol procedure.

These standardized RNA isolation procedures guarantee high-quality nondegraded RNA. RNA samples were quality checked by identification of $18 \mathrm{~S}$ rRNA and $28 \mathrm{~S}$ rRNA peaks via the Agilent 2100 Bioanalyzer platform (Agilent Technologies, Santa Clara, CA, USA). The yields were $8-15 \mu \mathrm{g}$ and RNA integrity number was between 7.2 and 10. According to published data, RNA with RNA integrity number of $>6$ is of sufficient quality for gene expression profiling experiments as well as microRNA microarray experiments. ${ }^{18}$

\section{Quantitative real-time RT-PCR for expression of hTERT}

Complementary DNA (cDNA) was synthesized from $\sim 2 \mu \mathrm{g}$ RNA using the Transcriptor High Fidelity cDNA Synthesis Kit (Roche, Berlin, Germany). Relative concentrations of cDNA samples were evaluated by quantitative reverse transcriptase-PCR (RT-PCR) of $\beta$-actin performed on Bio-Rad iQ5 Real Time PCR System (Bio-Rad, Hercules, CA, USA), followed by analysis of the gene expression of hTERT using the same procedure. To amplify the cDNA $300 \mathrm{ng}$ aliquots of reverse-transcribed cDNA were subjected to PCR amplification in $25 \mu \mathrm{l}$ containing $12.5 \mu \mathrm{l}$ of $2 \times$ FastStart SYBR Green Master (Roche) and $0.28 \mu \mathrm{M}$ primers. The following primes were used:

hTERT-F: $5^{\prime}$-GAGAACAAGCTGTTTGCGGG-3'

hTERT-R: 5'-AGCCATACTCAGGGACACCT- $3^{\prime}$

$\beta$-actin-F: $5^{\prime}$-AGCACAGAGCCTCGCCTTTGCC- ${ }^{\prime}$

$\beta$-actin -R: $5^{\prime}$-ACGAGCGCGGCGATATCATCATC- $3^{\prime}$.

The reaction conditions were initial denaturation at $95^{\circ} \mathrm{C}$ for $10 \mathrm{~min}$, followed by 40 cycles of denaturation at $95^{\circ} \mathrm{C}$ for $15 \mathrm{~s}$, annealing and extension at $61{ }^{\circ} \mathrm{C}$ for $30 \mathrm{~s}$. The melting curve analysis was conducted increasing each cycle by $0.5^{\circ} \mathrm{C}$ starting from $58.0^{\circ} \mathrm{C}$ to $95^{\circ} \mathrm{C}$, each one of $10 \mathrm{~s}$.

Quantitative analysis was performed using the iQ5 Optical System Software (Bio-Rad). Duplicate quantification tests were performed, and the average was calculated for each sample. Each run of amplification included cDNA synthesized from RNA of MO 3.13 (an immortalized human oligodendroglia

Table 2 Group 2 patient characteristics of study subjects from Monaldi Hospital, Naples, Italy

\begin{tabular}{lcc} 
Characteristics & SALS patients $(\mathrm{n}=10)$ & Controls $(\mathrm{n}=10)$ \\
\hline Age (years) & $62.2 \pm 8.4$ & $62.1 \pm 8.1$ \\
Gender & 5 & 5 \\
$\quad$ Males $(n)$ & 5 & 5 \\
$\quad$ Females $(n)$ & $12.5 \pm 8.9$ & - \\
Mean length of the disease (months) & & - \\
Onset of the disease (\%) & $30 \%$ & - \\
$\quad$ Bulbar & $70 \%$ & \\
Limb & &
\end{tabular}

Abbreviation: SALS, sporadic amyotrophic lateral sclerosis. 
cell line) used as a positive control and a negative control lacking cDNA. Amplification of the housekeeping gene $\beta$-actin was performed to standardize the amount of sample RNA. Relative quantification of gene expression was performed by the $2^{-\Delta \Delta \mathrm{ct}}$ method.

Real-time quantitative PCR for p53 and p21 mRNA quantification For real-time quantitative RT-PCR total RNA was extracted with Trizol reagent (Gibco BRL, Grand Island, NY, USA) according to the manufacturer's instructions, and the concentration was measured by ultraviolet spectrophotometry, and its quality was examined by formaldehyde denatured agarose gel electrophoresis.

The cDNA was synthesized from $\sim 2 \mu \mathrm{g}$ RNA using the Transcriptor High Fidelity cDNA Synthesis Kit (Roche). Relative concentrations of cDNA samples were evaluated by quantitative RT-PCR of $\beta$-actin performed on the Bio-Rad iQ5 Real Time PCR System (Bio-Rad), followed by analysis of the gene expression of p53 and p21 using the same procedure. To amplify the cDNA, $100 \mathrm{ng}$ aliquots of reverse-transcribed cDNA were subjected to PCR amplification in $25 \mu \mathrm{l}$ containing $12.5 \mu \mathrm{l}$ of $2 \times$ FastStart SYBR Green Master (Roche) and $0.28 \mu \mathrm{M}$ primers.

The following primes were used:

p21 F: 5'-CTGGGGATGTCCGTCAGAAC- $3^{\prime}$

p21 R: $5^{\prime}$-CATTAGCGCATCACAGTCGC- $3^{\prime}$

p53 F: 5'-CCATGAGCGCTGCTCAGATA- $3^{\prime}$

p53 R: $5^{\prime}$-TGGACAGTGCTCGCTTAGTG-3'

$\beta$-actin-F: $5^{\prime}$-AGCACAGAGCCTCGCCTTTGCC- $3^{\prime}$

$\beta$-actin-R: 5'-ACGAGCGCGGCGATATCATCATC-3'

Amplification protocols used for p53 and p21 expression analysis were: $95^{\circ} \mathrm{C}$ for $10 \mathrm{~min}$ followed by 40 cycles of denaturation at $95^{\circ} \mathrm{C}$ for $40 \mathrm{~s}$, annealing at $56{ }^{\circ} \mathrm{C}(\mathrm{p} 53), 54{ }^{\circ} \mathrm{C}$ or $55^{\circ} \mathrm{C}(\mathrm{p} 21)$ for $50 \mathrm{~s}$ and extension at $72{ }^{\circ} \mathrm{C}$ for $1 \mathrm{~min}$. The melting curve analysis was conducted increasing each cycle by $0.5^{\circ} \mathrm{C}$ starting from 54.0 to $95^{\circ} \mathrm{C}$, each one of $10 \mathrm{~s}$.

On each experiment, no-template controls and no-reverse transcribed controls were also performed at the same time. No signals were detected in no-template controls and no-reverse transcribed controls. The average threshold cycle for each sample was determined from triplicate reactions and the expression level was normalized to $\beta$-actin. The relative amount of mRNA was calculated by the $2^{-\Delta \Delta \mathrm{ct}}$ method.

\section{Terminal restriction fragment length analysis}

Mean telomere length and telomerase activity were measured quantitatively in genomic DNA from whole blood. Genomic DNA was prepared using Gentra Puregene Blood Kit (Quiagen, Valencia, CA, USA). The telomeres were measured using a real-time PCR method, as described by Cawthon. ${ }^{19}$ Telomere length values were measured in blood genomic DNA by a quantitative PCR assay that determines the relative ratio of telomere repeat copy number to single-copy gene copy number (T/S ratio) in sALS samples as compared with the reference DNA sample (DNA from healthy control patients).

\section{Statistical analysis}

All results shown are mean \pm s.d. of at least three separate experiments, measuring each parameter by triplicate $(n=3)$. Statistically significant differences were tested by one-way analysis of variance and, when the $F$-value was significant, by Student-Newman-Keuls test. The $P$-value of $<0.05\left(^{*}\right)$ was considered statistically significant. In addition, we computed Pearson's correlation coefficient to describe associations between measures.

\section{In situ hybridization (ISH) assay}

Following hydration, endogenous peroxidase activity was quenched for $1 \mathrm{~h}$ with $3 \% \mathrm{H}_{2} \mathrm{O}_{2}$ in phosphate-buffered saline (PBS), and then slides were briefly washed in PBS and postfixed for 15 min with $4 \%$ paraformaldehyde in $1 \times$ PBS, washed in PBS and then a 10-min acetylation step was performed in $0.1 \mathrm{M}$ triethanolamine buffer $\mathrm{pH} 8.0$, containing acetic anhydride to a concentration of $0.25 \%(\mathrm{v} / \mathrm{v})$. After two washes in $2 \times$ saline sodium citrate (SSC), proteinase $\mathrm{K}$ digestion was run as above. Then, sections are washed twice in PBS and once in $2 \times$ SSC. The prehybridization was run in 50\% formamide,
$5 \times$ SSC, $0.1 \mathrm{M}$ dithiothreitol (DTT), $100 \mu \mathrm{g} \mathrm{m}^{-1}$ Heparin, $250 \mu \mathrm{g} \mathrm{m}^{-1}$ transfer RNA, $250 \mu \mathrm{g} \mathrm{ml}^{-1}$ single-stranded DNA $0.5 \times$ Denhardt's solution made up in sterile water for $2 \mathrm{~h}$ and $30 \mathrm{~min}$ at $37^{\circ} \mathrm{C}$ in a humidified chamber. The hybridization mix was prepared by adding in the previously described hybridization buffer three oligonucleotides: (5'-DigTCATCCACCAAACG CAGGAGCA-3'Dig pos, 5'-DigN-TGTCAGAGATGACGCGCAGGA-3' Dig, $5^{\prime}$-DigN-AGCCACGAACTGTCGCATGTA-3' ${ }^{\prime}$ ig) to a final concentration of $200 \mathrm{ng} \mathrm{ml}^{-1}$ each. Slides were covered with parafilm and incubated overnight at $37^{\circ} \mathrm{C}$ in the humidified chamber. After hybridization, tissue sections were washed as follows: $5 \mathrm{~min}$ in $1 \times$ SSC and $10 \mathrm{~mm}$ DTT at room temperature, $2 \times 15 \mathrm{~min}$ in $1 \times \mathrm{SSC}$ and $10 \mathrm{~mm}$ DTT at $55^{\circ} \mathrm{C}, 2 \times 15 \mathrm{~min}$ in $0.5 \times \mathrm{SSC}$ and $10 \mathrm{~mm}$ DTT at $55^{\circ} \mathrm{C}, 2 \times 15 \mathrm{~min}$ in $0.5 \times$ SSC and $10 \mathrm{~mm}$ DTT at $55^{\circ} \mathrm{C}$, $1 \times 10 \mathrm{~min}$ in $0.5 \times \mathrm{SSC}$ and $10 \mathrm{~mm}$ DTT at room temperature.

The sections were incubated for $3 \mathrm{~h}$ at room temperature in blocking solution ( $1 \% \mathrm{w} / \mathrm{v}$ blocking reagent Roche, $5 \% \mathrm{v} / \mathrm{v}$ sheep serum in PBS) and then with the anti-digoxigenin, Fab fragments POD conjugated antibody (Roche) in a 1:400 dilution in blocking solution and incubated overnight at $4{ }^{\circ} \mathrm{C}$. Then, $2 \mathrm{~h}$ washes were performed in TNT buffer $(0.1 \mathrm{M}$ Tris $\mathrm{pH} 7.5$, $0.15 \mathrm{M} \mathrm{NaCl}, 0.05 \%$ Tween-20), and detection was run with TSAPLUS Cyanine 3 Fluorescence Kit (Perkin Elmer, Santa Clara, CA, USA) according to the instructions of manufacturer. The signals were visualized under Zeiss Confocal Microscope LSM 510 (Zeiss, Milan, Italy). The sections were counterstained with a nuclear dye, 4',6-diamidino-2-phenylindole.

\section{RESULTS}

\section{The hTERT expression}

In spinal cord from post-mortem sALS patients, hTERT expression fell compared with controls, showing a 96\% decrease (Figure 1a).

The hTERT expression was also detected in leukocytes from all the controls and sALS patients (Figure $1 \mathrm{~b}$ ). The sALS patients exhibited a significant messenger TERT decrease, a five- to six-fold decrease, in the expression of messenger TERT RNA transcripts compared with healthy controls.

\section{P53 and p21 expression}

P53 and p21 were detected in both leukocytes and post-mortem spinal cord from all ALS patients (Figure 2). P53 and p21 in both tissues from ALS patients have been found to be overexpressed as compared with controls $(P<0.05)$.

\section{Relationships between expression of p21, p53 and hTERT}

The expression of p53 was related to hTERT expression in ALS patients $(r=0.78, P=0.0001)$. The level of expression of $\mathrm{p} 21$ was also correlated with the level of hTERT mRNA $(r=0.81, P=0.0001)$ in ALS (Figure 3).

\section{Telomere length}

For measuring telomere length, real-time PCR assays were used in all leukocyte samples. Among the 50 sALS patients, the telomere length ratio $(\mathrm{T} / \mathrm{S})$ was significantly lower in the patients (15\%) compared with the 50 control subjects $\left({ }^{\star} P<0.05\right.$; Figure 4$)$. Therefore, this finding looks as a consequence that telomerase, the reverse transcriptase that elongates telomeres through the de novo synthesis of TTAGGG repeats, was more inactive in sALS patients.

\section{Telomere length and hTERT mRNA expression}

Telomere length was correlated to mRNA expression of hTERT in leukocytes from each group of patients. Significant positive correlations between the decrease in telomere length and the decrease of hTERT expression were found in both sALS patients $(r=0.786$; $P<0.001$; Figure 5a) and healthy controls $(r=0.705 ; P<0.001$; Figure 5b). 


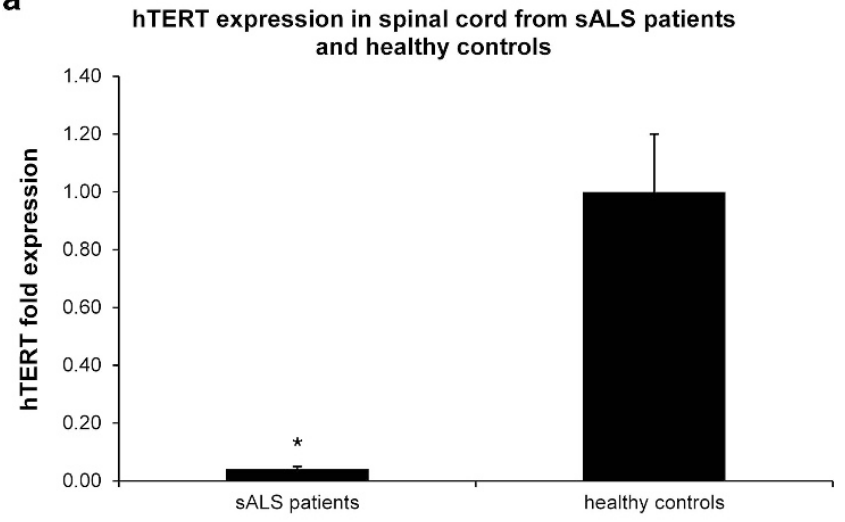

b

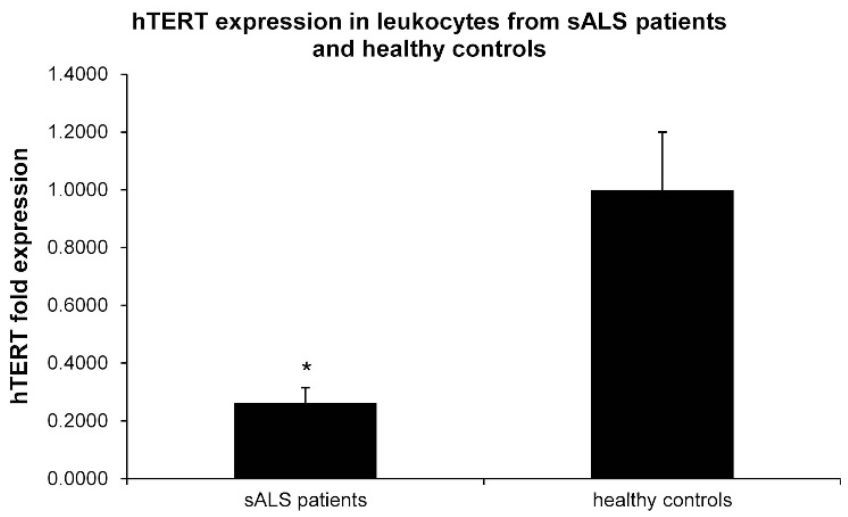

Figure 1 Human telomerase reverse transcriptase (hTERT) normalized messenger RNA (mRNA) expression in sporadic amyotrophic lateral sclerosis (sALS) patients and healthy controls. hTERT normalized mRNA expression in spinal cord (a) and leukocytes (b) from SALS patients and healthy controls. $\beta$-Actin expression was used for normalization. Data are mean ( \pm s.d.) of telomere lengths from sALS patients and healthy controls measured in triplicate. The asterisk indicates a significant difference between sALS patients with control group $\left({ }^{*} P<0.05\right)$.

\section{ISH assay}

The hTERT expression was further assayed by ISH for validation.

To confirm the decrease of hTERT expression in spinal cord in sALS patients, we examined spinal cord tissue samples collected at autopsy from patients with sALS $(n=3)$ and age-matched controls $(n=3)$. ISH demonstrated the nuclear localization of hTERT in the gray matter of the spinal cord (lumbar region (Figure 6). The sALS sample signal of hTERT-positive cells was strongly reduced as compared with the healthy controls.

The ISH results were consistent with real-time results, as it showed significantly decreased levels of hTERT expression in the spinal cord, a tissue directly related to ALS disease, by real-time PCR analysis.

\section{DISCUSSION}

Telomerase activity is considered to be absent (or at least undetectable) in postmitotic neurons. However, expression of telomerase has been reported in multiple neuronal subtypes such as rat hippocampal neurons and spinal cord motor neurons in vitro and cerebellar Purkinje neurons in vivo. ${ }^{20,21}$ Growing evidence suggests that telomerase, and its catalytic subunit TERT, may have antiapoptotic activity to countenance neuronal insult that could be exploited as a therapeutic target in neurodegeneration. In rat pheochromocytoma

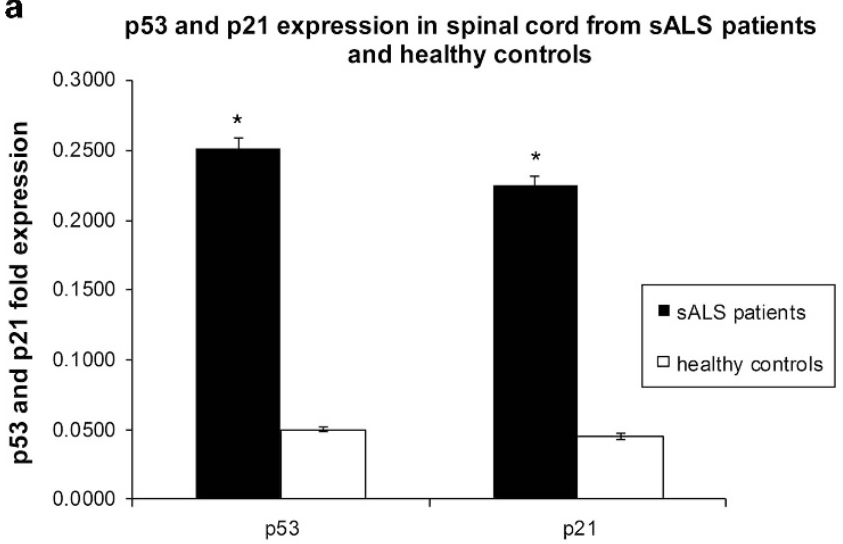

b

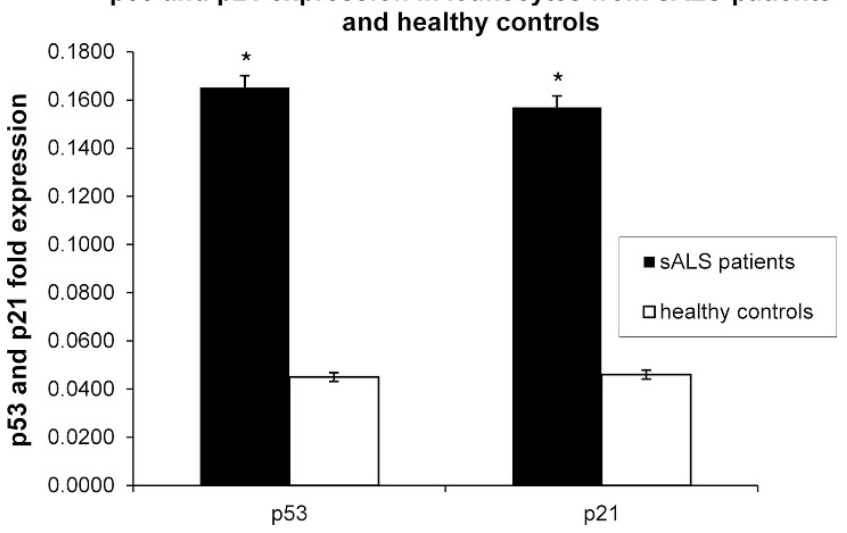

Figure 2 P53 and p21 normalized messenger RNA (mRNA) expression in sporadic amyotrophic lateral sclerosis (SALS) patients and healthy controls. P53 and p21 normalized mRNA expression in spinal cord (a) and leukocytes (b) from sALS patients and healthy controls. $\beta$-Actin expression was used for normalization. Data are mean ( \pm s.d.) of telomere lengths from sALS patients and healthy controls measured in triplicate. The asterisk indicates a significant difference between SALS patients with control group $\left({ }^{*} P<0.05\right)$.

\section{Correlation between spinal cord p53/hTERT and p21/hTERT expression in sALS patients}

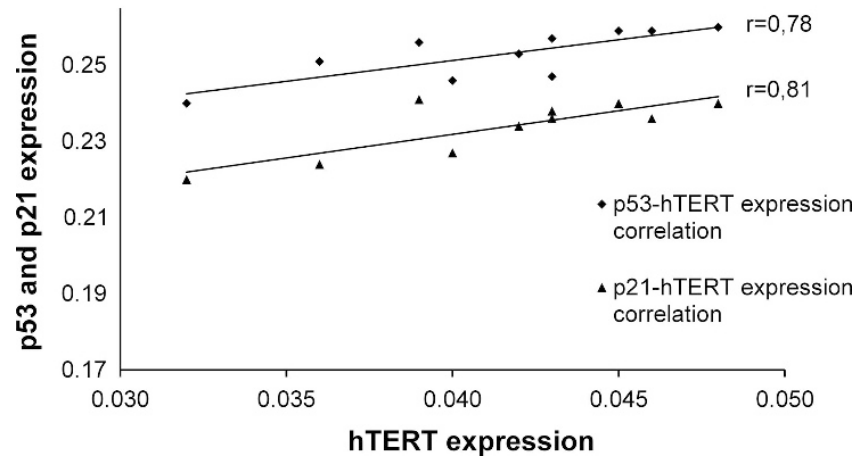

Figure 3 Correlation of p53 and p21 with human telomerase reverse transcriptase (hTERT) expression. Correlation of p53 and p21 with hTERT expression in spinal cord from sporadic amyotrophic lateral sclerosis (sALS) patients $(P<0.001)$.

PC12 cells (used widely as an in vitro neuronal model), TERT overexpression made these cells significantly more resistant to DNA damage by the topoisomerase inhibitors etoposide and 


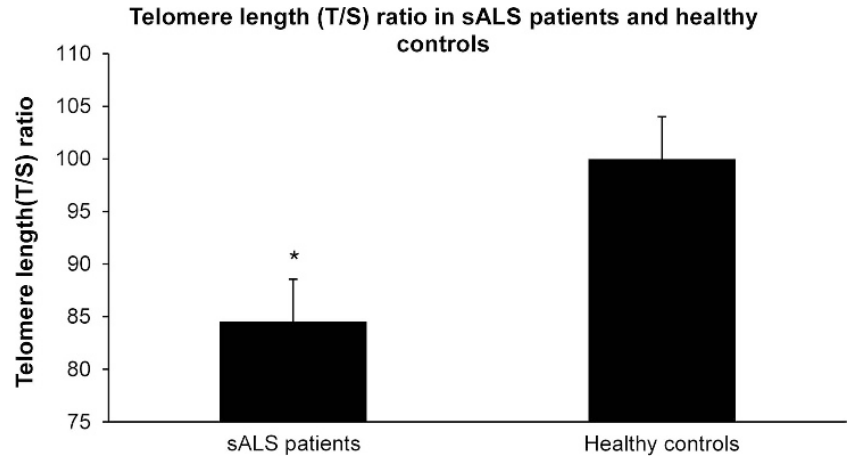

Figure 4 Quantitative estimation of the telomeres shortening rate between sporadic amyotrophic lateral sclerosis (SALS) patients and healthy controls. Telomere shortening rate in leukocytes from sALS patients and healthy controls. Data are mean ( \pm s.d.) of telomere lengths from 50 sALS patients and 50 controls measured in triplicate. The asterisk indicates a significant difference between sALS patients and healthy controls with ${ }^{*} P<0.05$.

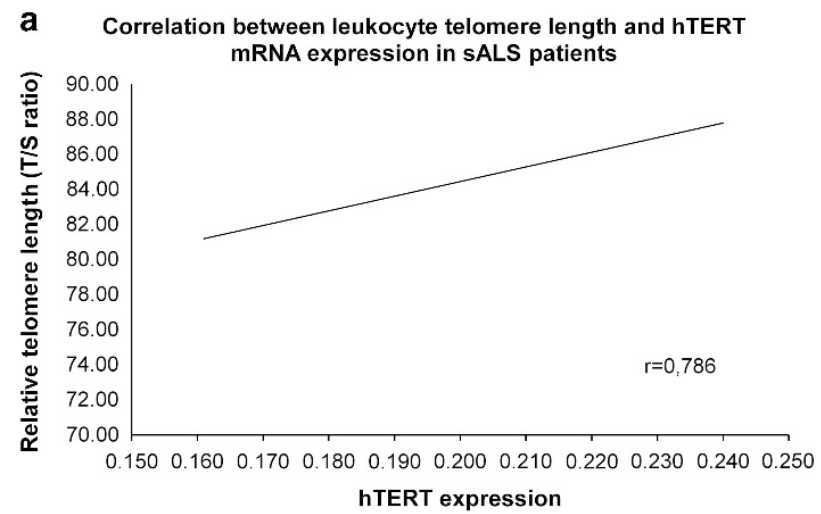

b Correlation between leukocyte telomere length and hTERT

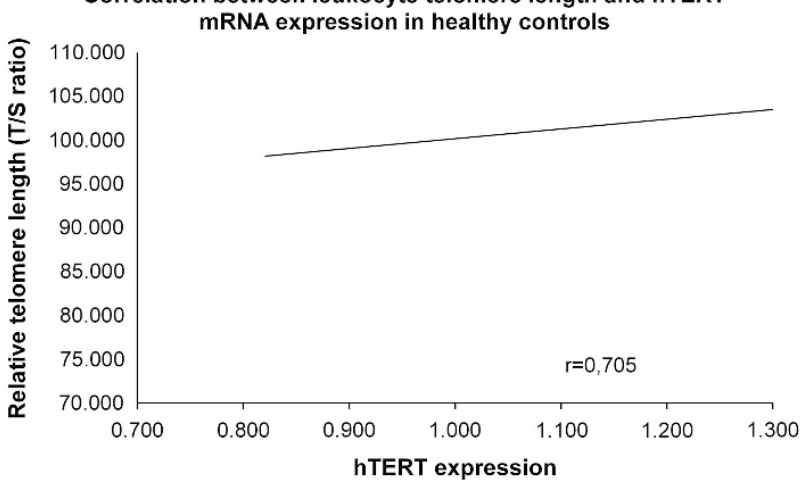

Figure 5 Correlation of telomere length with human telomerase reverse transcriptase (hTERT) expression. Correlation of telomere length with hTERT expression in leukocytes from (a) sporadic amyotrophic lateral sclerosis (sALS) patients and (b) healthy controls. $P<0.001$.

camptothecin. ${ }^{22}$ On the contrary, antisense knockdown of TERT in cultured hippocampal neurons increased their sensitivity to the same DNA-damaging agents. Neuroprotective roles for TERT have also been described in in vitro models of oxidative stress and excitotoxicity. ${ }^{23,24}$ Nevertheless, the mechanisms by which telomerase exerts antiapoptotic effects on neurons remain to be clarified.

In the current study, by real-time PCR assay, we detected a strong decrease of hTERT expression in post-mortem spinal cord from sALS
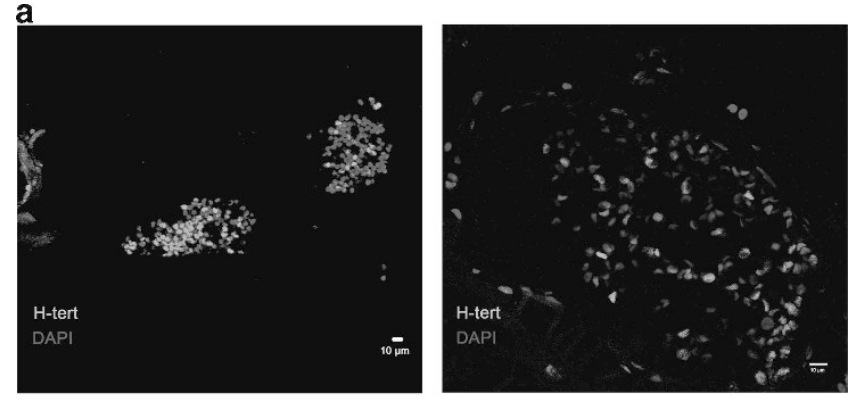

b
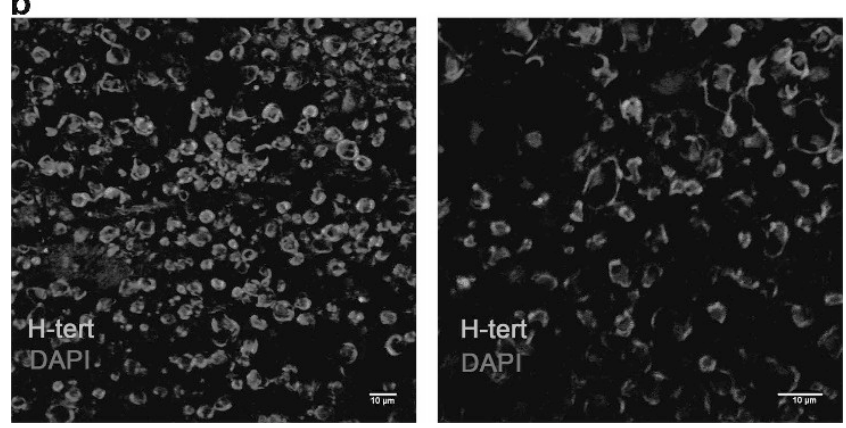

Figure 6 In situ hybridization on paraffin spinal cord sections. In situ hybridization on paraffin spinal cord sections from healthy controls (a) and amyotrophic lateral sclerosis (ALS) patients (b). A full color version of this figure is available at the Journal of Human Genetics journal online.

patients compared with healthy controls. As it has been suggested that the canonical function of telomerase is to support genomic stability and protective effects against neuronal insult, the lack of hTERT expression in sALS could be an additional requisite for neurodegeneration.

Beside, in situ experiments confirmed hTERT nuclear localization and underexpression in the gray matter of spinal cord tissue samples from sALS-autopsied patients.

Further evidence suggests that TERT may act via several mechanisms to suppress both the intrinsic and extrinsic pathways of apoptosis, ${ }^{25}$ and noncanonical, extratelomeric functions of telomerase may also contribute to cellular protection against oxidative damage.

TERT can suppress caspase activation in PC12 cells exposed to apoptotic insults. ${ }^{26}$ Recent findings suggest a role for direct or indirect actions of TERT with the tumor suppressor protein p53 in the antiapoptotic function of telomerase. $\mathrm{Li}$ et al. ${ }^{27}$ reported that the TERT-binding protein TEP-1 interacts directly with p53, and suggested that this interaction may inhibit telomerase activity. The p53 plays a role in many different models of neuronal apoptosis and has been involved in neuronal cell death. ${ }^{28,29}$ In ALS, motor neuron degeneration is associated with changes in the levels of $\mathrm{p} 53 .{ }^{30}$ Indeed, elevated levels of p53 have been identified in ALS spinal cord motor neurons, and therefore $\mathrm{p} 53$ has been linked to an apoptotic mode of cell death in ALS spinal cord motor neurons. ${ }^{31}$ In the present research, we detected an increased p53 expression level in both spinal cord and leukocytes from sALS patients, confirming the results obtained previously. ${ }^{30,31}$

The correlations between the expressions of p21, p53 and hTERT in ALS have not been investigated yet.

We found out altered levels of p53 and pro-apoptotic protein p21 in both sALS leukocytes from alive patients and post-mortem spinal cord tissues. Interestingly, p53 and p21 mRNA levels were also 
increased in ALS leukocytes from patients at 6-12 months of onset disease. In normal physiologic conditions, p53 has a very short half-life and is rapidly degraded. The presence of elevated levels of p53 in ALS patients is significantly correlated with p21, as well as hTERT expression.

It is possible that p53 transcripts are stabilized in ALS patients through p21 pathway, and modulation of p53-dependent mechanisms might contribute to TERT function. Repression of hTERT expression by endogenous p 53 has been already demonstrated to be indirect and mediated by $\mathrm{p} 21 .^{32}$

Telomerase is responsible for maintaining telomere length by the addition of TTAGGG sequences to the chromosome end. Interestingly, in leukocytes from sALS patients, compared with healthy control group, we identified reduced hTERT expression as well as shorter telomeres.

Recent studies have linked dysfunction of the telomeric complex to a variety of neurological disease states. Short telomeres, defects in several proteins involved in telomere maintenance (including TERT neurological disturbances including learning difficulties, mental retardation and microcephaly) have been reported in patients with dyskeratosis congenita. ${ }^{33}$ Significantly shorter leukocyte telomere length in patients with Alzheimer's disease as compared with agematched healthy controls has been reported. ${ }^{34}$ In sALS, we found that shortened telomere length is related to low level of hTERT expression, and therefore could be a consequential event; however, further investigation is needed to determine the role of telomere erosion in ALS.

In sALS, telomerase inhibition could be a pathogenetic contributor to neurodegeneration. A lack of telomerase activity does not protect neurons against a variety of insults. Indeed, noncanonical, extratelomeric functions of telomerase may also contribute to cellular protection against oxidative damage. ${ }^{35}$ The neuroprotective roles for telomerase have been described in PC12 cells (used widely as an in vitro neuronal model) and hippocampal neurons used as in vitro models of oxidative stress and excitotoxicity. 22,23

It has been shown that the low levels of TERT present in mature neurons ${ }^{24}$ might contribute to their vulnerability to various metabolic and oxidative insults. ${ }^{26}$ Beside, it has been suggested that telomerase induction might represent an endogenous compensatory mechanism to protect against ischemic injury in the brain. ${ }^{23}$

The beneficial effects of telomerase stimulation have been shown in the SOD1 transgenic mouse model of ALS. ${ }^{36}$ In this study, the authors used a novel triaryl compound, AGS-499, to induce expression and activity of telomerase in various regions of the central nervous system and spinal cord, delaying disease progression.

Taken together, these findings, together with our results, suggest that lack of telomerase may contribute to neuronal death in sALS.

If neurodegenerative disorders may result from abnormal apoptotic death of neurons and telomere and its associated proteins (for example, telomerase) may regulate neuronal response to oxidative stress and DNA damage, a better understanding of the role of telomerase and other components of the telomeric complex may lead to novel therapeutic approaches to attenuate neuronal injury. Further research should be aimed to address these important issues.

\section{CONFLICT OF INTEREST}

The authors declare no conflict of interest.
1 Nakamura, T. M., Morin, G. B., Chapman, K. B., Weinrich, S. L., Andrews, W. H. Lingner, J. et al. Telomerase catalytic subunit homologs from fission yeast and human Science 277, 955-959 (1997).

2 Blasco, M. A. Telomeres and human disease: ageing, cancer and beyond. Nat. Rev. Genet. 6, 611-622 (2005).

3 Yoo, J., Park, S. Y., Kang, S. J., Kim, B. K., Shim, S. I. \& Kang, C. S. Expression of telomerase activity, human telomerase RNA, and telomerase reverse transcriptase in gastric adenocarcinomas. Mod. Pathol. 16, 700-707 (2003).

4 Vogelstein, B., Lane, D. \& Levine, A. J. Surfing the p53 network. Nature 408 307-310 (2000)

5 Kusumoto, M., Ogawa, T., Mizumoto, K., Ueno, H., Niiyama, H., Sato, N. et al. Adenovirus-mediated p53 gene transduction inhibits telomerase activity independent of its effects on cell cycle arrest and apoptosis in human pancreatic cancer cells. Clin. Cancer Res. 5, 2140-2147 (1999).

6 Beausejour, C. M., Krtolica, A., Galimi, F., Narita, M., Lowe, S. W., Yaswen, P. et al. Reversal of human cellular senescence: roles of the p53 and p16 pathways. EMBO J. 22, 4212-4222 (2003).

7 Tao, Q., Lv, B., Qiao, B., Zheng, C. Q. \& Chen, Z. F. Immortalization of ameloblastoma cells via reactivation of telomerase function: phenotypic and molecular characteristics. Oral Oncol. 45, 239-244 (2009).

8 Liu, X., Dakic, A., Chen, R., Disbrow, G. L., Zhang, Y., Dai, Y. et al. Cell-restricted immortalization by human papillomavirus correlates with telomerase activation and engagement of the hTERT promoter by Myc. J. Virol. 82, 11568-11576 (2008)

9 Haferkamp, S., Tran, S. L., Becker, T. M., Scurr, L. L., Kefford, R. F. \& Rizos, H. The relative contributions of the $\mathrm{p} 53$ and pRb pathways in oncogene induced melanocyte senescence. Aging (Albany, NY) 1, 542-556 (2009).

10 Ohtani, N., Yamakoshi, K., Takahashi, A. \& Hara, E. Realtime in vivo imaging of p16Ink4a gene expression: a new approach to study senescence stress signaling in living animals. Cell. Div. 5, 1 (2010).

11 Zhou, J., Ding, D., Wang, M. \& Cong, Y. S. Telomerase reverse transcriptase in the regulation of gene expression. BMB Rep. 47, 8-14 (2014).

$12 \mathrm{Ly}, \mathrm{H}$. Genetic and environmental factors influencing human diseases with telomere dysfunction. Int. J. Clin. Exp. Med. 2, 114-130 (2009)

13 Nelson, N. D. \& Bertuch, A. A. Dyskeratosis congenita as a disorder of telomere maintenance. Mutat. Res. 730, 43-51 (2012).

14 Jaskelioff, M., Muller, F. L., Paik, J. H., Thomas, E., Jiang, S., Adams, A. C. et al Telomerase reactivation reverses tissue degeneration in aged telomerase-deficient mice. Nature 469, 102-106 (2011).

$15 \mathrm{Zhu}, \mathrm{H}$., Fu, W. \& Mattson, M. P. The catalytic subunit of telomerase protects neurons against amyloid beta-peptide-induced apoptosis. J. Neurochem. 75, 117-124 (2000)

16 De Felice, B., Guida, M., Guida, M., Coppola, C., De Mieri, G. \& Cotrufo, R. A. miRNA signature in leukocytes from sporadic amyotrophic lateral sclerosis. Gene $\mathbf{5 0 8}, \mathbf{3 5}-40$ (2012).

17 Tichon, A., Gowda, B. K., Slavin, S., Gazit, A. \& Priel, E. Telomerase activity and expression in adult human mesenchymal stem cells derived from amyotrophic latera sclerosis individuals. Cytotherapy 11, 837-848 (2009).

18 Bissels, U., Wild, S., Tomiuk, S., Holste, A., Hafner, M., Tuschl, T. et al. Absolute quantification of microRNAs by using a universal reference. RNA 15, 2375-2384 (2009).

19 Cawthon, R. M. Telomere measurement by quantitative PCR. Nucleic Acids Res. 30, e47 (2002).

20 Eitan, E., Tichon, A., Daniel, G. \& Priel, E. Telomerase expression in adult and old mouse Purkinje neurons. Rejuvenat. Res. 15, 206-209 (2012).

21 Niu, C. \& Yip, H. K. Neuroprotective signaling mechanisms of telomerase are regulated by brain-derived neurotrophic factor in rat spinal cord motor neurons. J. Neuropathol. Exp. Neurol. 70, 634-652 (2011).

$22 \mathrm{Lu}, \mathrm{C}$., Fu, W. \& Mattson, M. P. Telomerase protects developing neurons against DNA damage-induced cell death. Brain Res. Dev. Brain Res. 131, 167-171 (2001).

23 Kang, H. J., Choi, Y. S., Hong, S. B., Kim, K. W., Woo, R. S., Won, S. J. et al. Ectopic expression of the catalytic subunit of telomerase protects against brain injury resulting from ischemia and NMDA-induced neurotoxicity. J. Neurosci. 24 1280-1287 (2004).

24 Fu, W., Begley, J. G., Killen, M. W. \& Mattson, M. P. Anti-apoptotic role of telomerase in pheochromocytoma cells. J. Biol. Chem. 274, 7264-7271 (1999).

$25 \mathrm{Li}$, J., Tang, B., Qu, Y. \& Mu, D. Telomerase reverse transcriptase: a novel neuroprotective mechanism involved in neonatal hypoxic-ischemic brain injury. Int. J. Dev. Neurosci. 29, 867-872 (2011).

26 Fu, W., Killen, M., Pandita, T. \& Mattson, M. P. The catalytic subunit of telomerase is expressed in developing brain neurons and serves a cell survival-promoting function. J. Mol. Neurosci. 14, 3-15 (2000).

27 Li, H., Cao, Y., Berndt, M. C., Funder, J. W. \& Liu, J. P. Molecular interactions between telomerase and the tumor suppressor protein p53 in vitro. Oncogene 18, 6785-6794 (1999).

28 Plesnila, N., von Baumgarten, L., Retiounskaia, M., Engel, D., Ardeshiri, A., Zimmermann, R. et al. Delayed neuronal death after brain trauma involves p53-dependent inhibition of NF-kappaB transcriptional activity. Cell Death Differ. 14, 1529-1541 (2007).

29 Yamaguchi, A, Taniguchi, M, Hori, O, Ogawa, S, Tojo, N, Matsuoka, N et al. Peg3/Pw1 is involved in p53-mediated cell death pathway in brain ischemia/hypoxia. J. Biol. Chem. 277, 623-629 (2002). 
30 Martin, L. J. p53 is abnormally elevated and active in the CNS of patients with amyotrophic lateral sclerosis. Neurobiol. Dis. 7(6 Pt B), 613-622 (2000).

31 Ranganathan, S. \& Bowser, R. p53 and cell cycle proteins participate in spinal motor neuron cell death in ALS. Open Pathol. J. 4, 11-22 (2010).

32 Shats, I., Milyavsky, M., Tang, X., Stambolsky, P., Erez, N., Brosh, R. et al. p53-dependent down-regulation of telomerase is mediated by p21waf1. J. Biol. Chem. 279, 50976-50985 (2004).

33 Nelson, N. D. \& Bertuch, A. A. Dyskeratosis congenita as a disorder of telomere maintenance. Mutat. Res. 730, 43-51 (2012).
34 Thomas, P., O'Callaghan, N. J. \& Fenech, M. Telomere length in white blood cells, buccal cells and brain tissue and its variation with ageing and Alzheimer's disease. Mech. Ageing Dev. 129, 183-190 (2008).

35 Ahmed, S., Passos, J. F., Birket, M. J., Beckmann, T., Brings, S., Peters, H. et al. Telomerase does not counteract telomere shortening but protects mitochondrial function under oxidative stress. J. Cell Sci. 121(Pt 7), 1046-1053.

36 Eitan, E., Tichon, A., Gazit, A., Gitler, D., Slavin, S. \& Priel, E. Novel telomerase increasing compound in mouse brain delays the onset of amyotrophic lateral sclerosis. EMBO Mol. Med. 4, 313-329 (2012). 